\title{
Application of Plastic wood material in the Artistic Modeling Design of Conservation Landscape
}

\author{
Sun xing \\ Media and Art College, Nanjing University of Information \&Technology, China, Nanjing, Jiang Su \\ sunxing1227@163.com
}

Keywords: Conservation Landscape, Plastic Wood, Artistic Modeling, Design

Abstract. In this paper, the new use of new materials for conservation landscape, especially wood plastic composites, is used as a starting point. The principles and strategies for the application of new materials in contemporary urban landscape are analyzed, and the artistic modeling design and example of plastic wood materials are introduced. The application method of new materials with guiding significance for practice is proposed.

\section{Introduction}

In the modern era of rapid economic development, the earth, wood, metal, water and other materials used by humans in landscape design have consumed increasing energy, and the destruction of the environment has also forced humans to reflect on the protection of the environment. The conservation landscape design is a sustainable landscape design method, and it is one of the important ways to realize the conservation landscape greening. The traditional garden gardening materials are mainly water, Stone, plants, etc.. Now the economical landscape design has expanded the idea of the use of landscape materials. Many artificial materials and modern high-tech materials have also been used in large quantities. Soft materials such as glass, transparent plastics, synthetic fibers, and rubber are rich in the life and dynamic sense of organic matter. Brings a fresh feel to the landscape design, The tree array pools and composite flower beds that can be seen everywhere in modern garden landscapes use new environmental protection materials, plastic wood materials. This material can greatly reduce the utility of wood and can recycle and use low-cost used wood. Believing that this will be a new reform of the timber market, which will promote the replacement of wood, and that the application of this new environmentally friendly material will fully meet the requirements of the Central Government in recent years for the rational and efficient recycling of abandoned wood, It is of great significance for the creation of economical society and the development of circular economy.

Plastic wood material is a new type of material made of wood fiber or plant fiber, which is combined with thermoplastic resin or other materials after pretreatment. It has high mechanical strength, high impact resistance, and thermal scalability. Water absorption is smaller than that of wood. Good size stability, wear resistance, chemical corrosion resistance, not afraid of moths, non-flammable, dual processing properties of wood and plastic. During the Shanghai World Expo, the outer wall of the Dutch Pavilion was made of 25,000 pieces of plastic wood composite materials made of waste paper and plastic. Plastic wood composite materials are not only used in the fields of benches, corridors, railings, etc. in landscape landscapes, but also have very important applications in garden sculptures. During the preparations for the Beijing Olympic Games, The four reliefs of Mei, Lan, Zhu and Ju, which are exclusively owned by Guofeng Wood Plastics Company and provide 250 $\times 20$ wood plastic plates, have become the most beautiful scenery in the Olympic Village model project with their exquisite carving techniques and extraordinary creativity. line.

2、Landscape Materials -- Selection and Design Principles of Plastic Wood

A successful landscape not only needs to meet people's physiological needs, but also can meet people's psychological needs. Man's satisfaction with environmental physiological needs is embodied in the realization of the use value, and the satisfaction of the psychological needs of the environment includes many aspects. With the changes and developments of the times, the psychological needs of people continue to change. In the landscape mainly reflected in the following aspects: ornamental 
value, ecological value and cultural value. And these values are mainly reflected through the successful application of landscape engineering materials.

The embodiment of the use value of artificially shaping the environment mainly depends on the physical properties of the materials, because the selected materials are used in complex environments and require certain hardness, strength, wear resistance, water resistance, light resistance and oxidation, and so on; The realization of ornamental value mainly depends on the sensory properties of the material, including color, texture, size, shape, texture, and texture, as well as the combination of materials. Therefore, the choice of emerging environmental shaping materials, including plastic wood, can not be limited to the original design techniques. Today, with the rapid development of science and technology and the frequent exchange of information, It is necessary to have certain principles and methods to recognize the new type of environmental shaping engineering materials, so that it can be used rationally in landscape design, so that the environment can develop synchronously with the times. In the selection and design of plastic wood materials, we should pay attention to several basic principles:

\section{(1) Safety Principles}

Landscape is a region closely related to human activities, the selection and application of plastic wood materials are considered from two aspects: the firmness of the materials and the safety of the materials themselves. After considering the construction and installation conditions, the strength of the material determines the quality of the environmental structure. Whether the selected material can resist the light environment, water and humidity environment, thermal environment, acid and alkali corrosion environment and other tests is an important basis for whether the material can pass the safety principle. On the other hand, some materials themselves also have certain security risks, and these issues must be considered when choosing.

(2) Functional principles

The various facilities in the landscape have a high frequency of use. Therefore, the materials used must be able to meet the basic functional needs. When selecting plastic wood materials in environmental molding, priority should be given to the purpose of the design and the use of specific uses. For example, in the selection of ground plastic wood paving materials, consideration is given to the low weight bearing, the need for Anti-Slip measures, and the prevention of wear caused by human walking. Usually, plastic wood plates with empty, high wear resistance parameters, and rough sugar and texture treatment are used. Therefore, different choices and juices should be made according to different landscapes to meet the functional requirements. Urban habitat environmental services are aimed at people. In the process of use, environmental engineering must have a definite use value, and the materials used must be able to meet the basic needs of people when they are used.

(3) Coordination with the environment Principles

The shaping of the modern landscape is a space that has the use value and the aesthetic value that relies on the human wisdom and the modern design concept to carry out reasonable planning. The materials chosen to construct the modern urban habitat environment must be able to integrate well with the surrounding environment. The new materials and traditional materials manufactured by modern science and technology are integrated into the environmental art design. If they can be well integrated with local cultural atmosphere, they can form a unique regional environmental engineering.

(4) Aesthetic, economic and ecological principles

The ecological requirements of the modern environment are mainly reflected in "local conditions". The products of plastic wood materials are rich and varied, but the appearance, texture, strength, and use conditions of each type of plastic wood product have certain characteristics. In the process of modern landscape shaping, it is necessary to select the most suitable plastic wood materials according to specific environmental conditions and use functions, and ensure that the aesthetic and functional aspects are equally emphasized.

(5) Feasibility of construction Principles

The construction feasibility of materials is a key point in environmental molding engineering. Under the premise that the selected materials can be made to ensure the supply, there is a close 
relationship between the construction conditions and the selection of materials in the area where the environmental molding engineering is located. For example, the requirements for the environmental and climatic conditions of the material construction site, such as temperature, humidity, or other changes, affect the construction effect of the material; The solar well-being caused by geographical location affects the aging of materials and the degradation of colors; Secondly, the construction machinery conditions and the construction personnel's own professional skills and other factors should be fully considered to ensure the quality of the construction.

3、Artistic Design of Plastic Wood Materials

Traditional plastic wood materials are mainly used in packaging materials, or as building materials for outdoor landscapes. Mainly used in landscape design of centralized paving boards(trails, steps, hydrophilic platforms, landscape balconies, etc.), guardrails(guardrails, railings, wooden fences, escalators, etc.), sketches(outdoor seats, tables, flower stands, outdoor swings, Flower beds, flower pots, flower boxes, etc.), garden auxiliary facilities garbage cans, signs, etc.) and garden buildings(wooden houses, wooden pavilions, wooden villas, wooden activity rooms, etc.) in the courtyard wood structure all wood plastic products can be used instead of solid wood, plastic products. However, with the continuous progress of society and the continuous improvement of science and technology, people gradually develop the artistic modeling of plastic wood materials and develop new functions of plastic wood materials in daily life.

The artistic modeling design of plastic wood materials can be manufactured by molding or injection molding. When molding, the plastic wood composite materials are first placed into the mold cavity, and the mold is closed, and the material is changed under the action of heat or pressure. In the mold, a shape that is consistent with the shape of the mold is molded. Using this molding method, an organic surface can be created, and the pattern texture of the surface can be processed at one time. Injection molding process is the injection of molten wood plastic composites into the mold cavity with high pressure, and after cooling and demoulding, it is made into a product that is consistent with the shape of the mold. The injection molding process can be used to obtain a rich variety of product shapes, not only for the manufacture of complete furniture., It is more suitable for the manufacture of furniture parts with complex surfaces. However, since the fluidity of the plastic melt is significantly lower than that of the homogenous plastic, the injection molding and even molding of the plastic wood is difficult and needs to be further strengthened.

In addition, plastic wood embossed sculpture technology, laser sintering technology, 3D printing technology can also be used to create landscape artistic modeling structure, especially for the manufacture of exquisite indoor and outdoor landscape decoration. However, at present, the cost of artistic modeling based on laser sintering and 3D printing technology is relatively high, and it is still difficult to apply in large-scale landscape applications.

4、Introduction of Concrete Examples of Artistic Design of Plastic Wood Materials

The author has studied the modeling design of plastic wood materials for many years, and innovatively proposed two kinds of design of plastic wood flooring, from the utility model patent "an outdoor foot massage plastic wood floor" and "a plastic wood floor with V-support". The first design is to put forward innovative applications for the modeling of plastic wooden floors. Traditional city parks have more massage paths made of goose-soft stone. The public stimulates foot acupuncture points by walking barefoot on goose-soft stone to promote the body's metabolic function. The purpose of protecting the health of the body by excluding wastes and toxins that accumulate in the body. However, there are obvious defects in the goose soft stone massage road laid in the park. For example, the loss and damage of goose soft stone will cause physical damage to the exercise public, and the massage road of

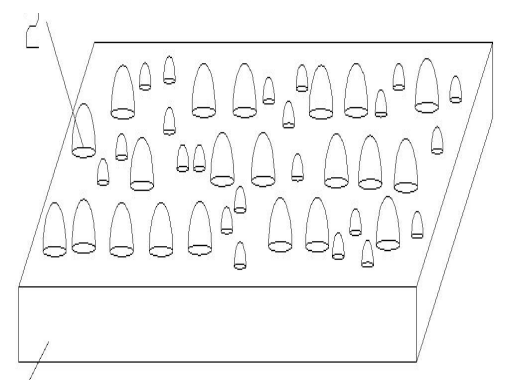

Fig. 1 uneven plastic wood floors

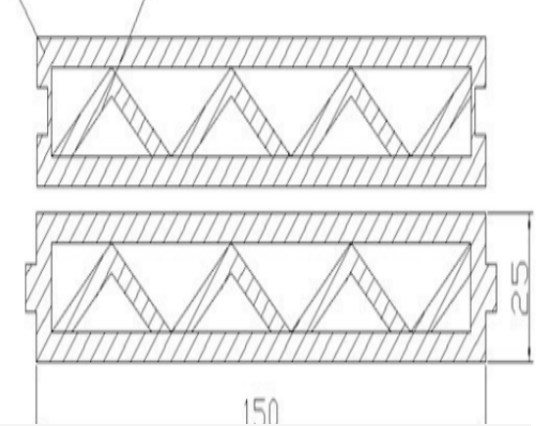

Fig.2 V-shapedsupported plastic wood floors 
stone material is difficult to repair. It is very difficult to maintain cleaning.

The Chinese patent ZL97228529.6 discloses a simulated Pebble pavement fitness felt, which achieves the effect on foot massage by sticking a simulated Pebble on the carpet. However, this paved pavement requires a large amount of felt cost, and the simulation Pebble strength is poor and easy to damage. The author proposes to prepare a kind of outdoor foot massage plastic wood floor. As shown in Figure 1, the surface of the wood is uneven and has dense protrusions. This kind of plastic wood material is laid into an outdoor massage road. Not only can it achieve the purpose of stimulating acupuncture points and exercising the body, but also the plastic wood material is environmentally friendly, energy-efficient, and durable. Even if it is damaged during use, it is very easy to replace. This plastic wood floor is arranged on the ground of an outdoor public space such as a park. People can walk barefoot or wear thin socks on the foot massage plastic wood road arranged above. The acupoints on the foot can be stimulated by the surface protrusion of the foot plastic wood floor. Through the uneven placement of prominent points can achieve different degrees of strength to increase the sensation of different acupoints on the foot, accelerate the body's metabolism, and play a health care role in the human body.

The second design is an innovative design of plastic wood flooring and the preparation of a plastic wood flooring with V-shaped support. As shown in Figure 2, the advantage of the utility model is that the plastic wood flooring adopts a hollow structure and reduces the weight of the floor. Reduced the raw materials for floor processing, while the V-shaped support structure designed by the hollow plastic wood flooring allows the entire plastic wood flooring to carry greater pressure. The existing size of the market is $150 \mathrm{~mm}$ wide $* 25 \mathrm{~mm}$ thick. The common four-hole hollow plastic wood bending strength is approximately $40 \mathrm{Mpa}$, The bending strength of the $\mathrm{V}$-shaped plastic wood floor of the utility model can reach $50 \mathrm{Mpa}$, the performance can be improved by $25 \%$, the bending strength of the plastic wood can be improved, and the practicality of the hollow plastic wood floor can be improved.

5、 tag

Modern landscape design with the improvement of people's ideological understanding, people not only require the urban environment to be practical, in its spatial sense, the design of the appearance of personality, as well as the expression of the spirit of the pursuit of the home, This pursuit is also reflected in the materials that create a modern environment atmosphere; In the process of application, plastic wood materials should pay attention to safety, functionality, coordination with the environment, meet the requirements of beauty, economy, ecology, etc..

\section{Acknowledgements}

This work was financially supported by 2016 Jiangsu University Philosophy and Social Science Research"2016SJD760008"; 2015 Jiangsu Provincial Social Science Applied Research Excellence Project "15SYC-035"

\section{References}

[1] Davide.Pendlenton, Thereas A. Hoffard. Durability of anextruded HDPE/wood composite. ForestProduct sjoumal,2002,52(6):21 27

[2] Madison Wl, Boratesas Fungicides in WPC. $7^{\text {th }}$ International Conference on Woodfiber-Plastie ComPosites. May19 20,2003

[3] Jennifer Markarian. Wood-Plastic Composites Need Protection, Too[J],Plastics Engineering,2007,63(7):12 16

[4]Jiang Dong. Analysis of the application of building materials in the landscape[ M] Beijing: Beijing Forestry University, 2005.

[5] Chengzhengwei.dulong, Zhang Qun compiled. Landscape construction engineering materials and construction[M].Beijing:Chemical Industry Press, 2009 
[6] Tahara, Yangdongdan. Design and application of environmental art decorative materials[ M] . Beijing: China Electric Power Press, 2008 